\title{
Myocarditis Associated with Coronavirus Disease 2019 (COVID-19) in a Pediatric Patient
}

\author{
Filippo M. Salvini ${ }^{*}$, Valentina Giuli ${ }^{2}$, Elisabetta F. Stacul ${ }^{1}$, Chiara Moretti ${ }^{1}$, Alessandra Corato ${ }^{2}$, \\ Sara Santacesaria ${ }^{2}$, Gabriele Vignati², Costantino De Giacomo ${ }^{1}$
}

${ }^{1}$ Division of Pediatrics, Niguarda Hospital, Milan, Italy

${ }^{2}$ Division of Pediatric Cardiology, Niguarda Hospital, Milan, Italy

Email: ^filippomaria.salvini@ospedaleniguarda.it

How to cite this paper: Salvini, F.M., Giuli, V., Stacul, E.F., Moretti, C., Corato, A., Santacesaria, S., Vignati, G. and De Giacomo, C. (2020) Myocarditis Associated with Coronavirus Disease 2019 (COVID-19) in a Pediatric Patient. Advances in Infectious Diseases, 10, 226-232.

https://doi.org/10.4236/aid.2020.103022

Received: September 16, 2020

Accepted: October 23, 2020

Published: October 26, 2020

Copyright $\odot 2020$ by author(s) and Scientific Research Publishing Inc. This work is licensed under the Creative Commons Attribution-NonCommercial International License (CC BY-NC 4.0). http://creativecommons.org/licenses/by-nc/4.0/

\begin{abstract}
Viral infection is one of the most important causes of myocarditis both in adult and pediatric age. We describe acute myocarditis due to SARS-CoV-2 infection in an otherwise healthy 12 -years-old Caucasian boy. He was admitted because of a history of 2 days of fever, dry cough, diarrhea, abdominal pain and fatigue. In spite of a family history of COVID-19 patient strict contact, a first nasopharyngeal swab for SARS-CoV-2 RNA resulted negative. After 4 days, his clinical condition evolved, with the onset of interstitial pneumonia, as documented by chest tomography and tachycardia. Blood analysis showed an NTproBNP and TnT increasing, transthoracic echocardiography revealed normal left ventricle (LV) dimension, altered myocardial texture, diffuse hypokinesis and impaired left ventricular function. A nasopharyngeal swab tested positive for SARS-CoV-2 infection at day 6 after admission. Heart failure conventional treatment associated with heparin, glucocorticoids and Remdesivir were administered with progressive improvement of clinical and instrumental conditions. No residual heart damage was documented during the following 3 monthes cardiological outpatient follow-up. Although COVID19 infection has a milder course of disease in children than in adults, severe forms of the disease may occur and myocarditis should be carefully considered in the pediatric age.
\end{abstract}

\section{Keywords}

Coronavirus Disease 19, Children, Myocarditis, Remdesivir

\section{Introduction}

Coronavirus disease 2019 (COVID-19) is caused by a novel enveloped RNA beta-coronavirus, named Severe Acute Respiratory Syndrome Coronavirus 2 (SARS- 
CoV-2), which is responsible for the pandemic started on December 2019 in $\mathrm{Wu}$ han (China) and rapidly spread worldwide [1] [2].

The typical initial signs of the disease are dry cough, fever, dyspnea, fatigue and an interstitial pattern with ground-glass opacities on chest computed tomography (CT) scans; less frequently the disease presentation is characterized by diarrhea and abdominal pain [3].

COVID-19 in pediatric patients seems to be less severe than in adults and the infection becomes symptomatic only in a minority of the affected children (less than $2 \%$, as reported in literature); hospitalization due to pneumonia or other COVID-19 related complications are rare among children [4] [5]. According to the literature, cardiovascular involvement determining chest pain, impaired ventricular function, arrhythmias and heart failure has been reported in a minority of adult patients affected by SARS-CoV-2 infection [6]. Cardiac involvement in children has been described in case series in the context of multisystem inflammatory syndrome (MIS-C) in current or recent SARS-CoV-2 infection, possibly involving coronary artery [7].

Our report describes the occurrence of myocardial injury in a pediatric patient with COVID-19 diarrhea and pneumonia.

\section{Case Presentation}

An otherwise healthy 12-years-old Caucasian boy without a previous history of any cardiovascular or chronic disease, presented to the pediatric emergency department with fever, sporadic dry cough, diarrhea, abdominal pain and fatigue, lasting since the two previous days.

He denied dyspnea and chest pain. His cohabitant grandfather died for SARS$\mathrm{CoV}-2$ pneumonia 10 days before. His temperature was $38.5^{\circ} \mathrm{C}$, blood pressure $100 / 70 \mathrm{mmHg}$, heart rate $120 \mathrm{bpm}$, the oxygen saturation $100 \%$ without oxygen support. On chest auscultation there were diffuse wheezes. Blood tests revealed an increase in C-reactive protein levels (C-RP $10.7 \mathrm{mg} / \mathrm{dL}$ ) and Procalcitonin (PCT $2.5 \mathrm{ng} / \mathrm{mL}$ ) with normal blood cells count. A nasopharyngeal swab was performed with a negative result for SARS-CoV-2 on real-time reverse transcriptase-polymerase chain reaction assay. CT scan indicated initial interstitial pulmonary infection and antibiotic therapy with Ceftriaxone was started. On the $4^{\text {th }}$ day of admission, his clinical condition worsened, with persistence of high grade fever, fatigue, abdominal pain and severe diarrhea. A second nasopharyngeal swab still resulted negative; on the blood tests further increase in C-RP levels $(15.3 \mathrm{mg} / \mathrm{dL})$ and PCT $(6.3 \mathrm{ng} / \mathrm{mL})$ were documented, together with the onset of lymphopenia, elevation of $\mathrm{N}$-terminal pro-brain natriuretic peptide (NTproBNP) (1013 ng/l) and normal high-sensitive troponin T (TnT). A 12-lead electrocardiogram (ECG) showed a sinus tachycardia. Abdominal ultrasound showed adeno-mesenteritis, together with mild ascites. Transthoracic echocardiography revealed normal left ventricle (LV) dimension, altered myocardial texture, diffuse hypokinesis with an estimated LV ejection fraction (LVEF) of 
$40 \%$. Twenty-four hours later the blood pressure decreased to $80 / 40 \mathrm{mmHg}$, ECG showed $\mathrm{T}$-wave inversion in $\mathrm{V} 4$ and flat $\mathrm{T}$ in V5-V6 and in the limb leads (Figure 1), TnT increased (30 ng/l), LVEF decreased until 30\% and there was mild pericardial effusion (Figure 2). Therapy with inotropic agent (Milrinone) and diuretics (Furosemide and Spironolactone) for heart failure was started in association with steroids (Methylprednisolone $2 \mathrm{mg} / \mathrm{kg} \times 3$ days, then progressively

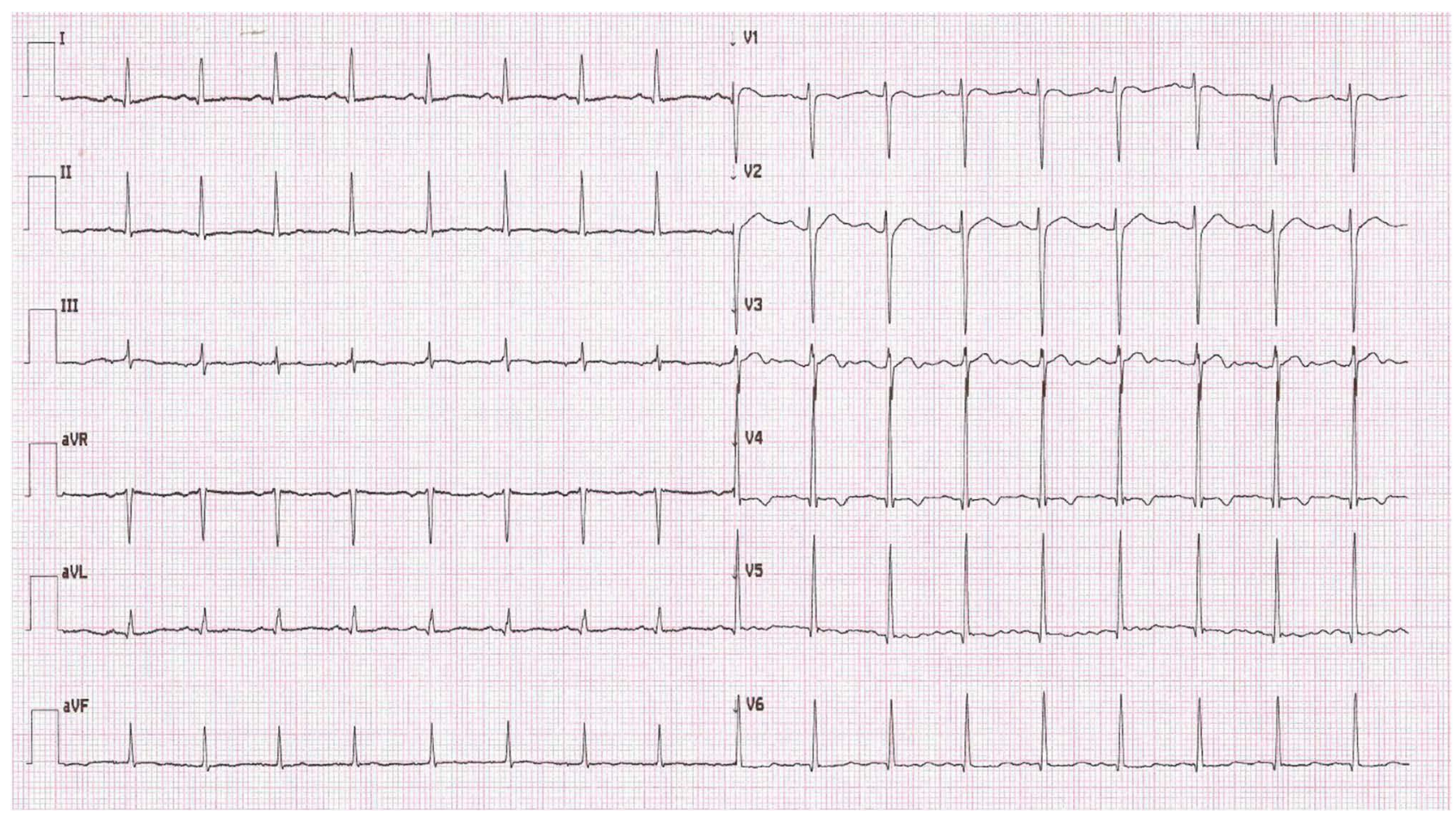

Figure 1. ECG: Electrocardiogram showing sinus tachycardia with T-wave inversion in V4 and flat T in V5 - V6 and in the limb leads.

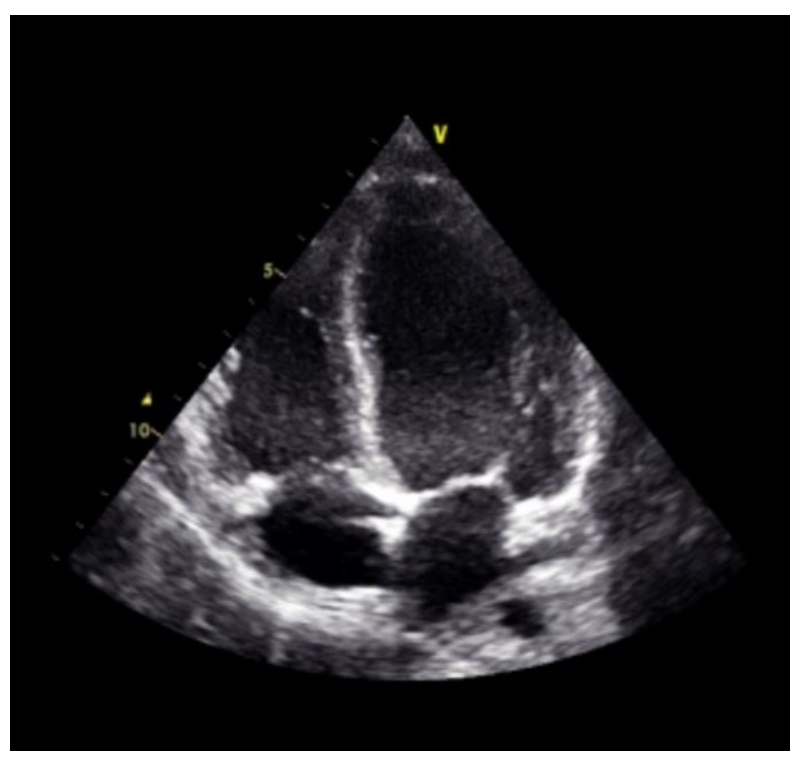

Figure 2. Echocardiography: 2D transthoracic echocardiogram (apical 4 chambers view) showing normal left ventricle dimension, altered myocardial texture and mild pericardial effusion. 
tapered) and Enoxaparine (4000 U) for the evidence of altered inflammatory and coagulation markers (Ferritin 1158 ng/mL; Fibrinogen 604 mg/dL; D-dimer 3.2 $\mathrm{mcg} / \mathrm{mL}$; international normalized ratio-INR 1.24).

A nasopharyngeal swab was repeated 6 days after admission testing positive for SARS-CoV-2, while search for common cardiotropic infectious agents yielded negative results. The abdominal-chest CT scan revealed bilateral basal pulmonary ground-glass opacities (Figure 3), pleural effusion and ascites.

Due to the progressive worsening of the patient's clinical symptoms and the SARS-CoV-2 documented infection, antiviral therapy with intravenous Remdesivir (compassionate use) was started on the $6^{\text {th }}$ day of admission for 10 consecutive days ( $200 \mathrm{mg}$ the first day, followed by $100 \mathrm{mg} /$ day). Local Ethical Committee authorization and parent consent were obtained before starting treatment.

The maximum value of TnT and of NTproBNP were $70 \mathrm{ng} / \mathrm{l}$ and 10,078 ng/L respectively (at $7^{\text {th }}$ day). In the following days there was an improvement of the clinical conditions: fever and diarrhea recovered 48 hours after steroids were started. Progressive normalization of the blood tests and substantial cardiac function improvement (LVEF 55\%) were also observed within the following 10 days. Inotropic agent was stopped on $8^{\text {th }}$ day of admission and Enalapril was started subsequently.

He was discharged after 20 days of hospitalization in good clinical condition and hemodynamic stability, after 2 SARS-CoV-2 negative nasopharyngeal swabs were obtained. No residual heart damage was documented during the following 3 monthes cardiological outpatient follow-up (cardiac magnetic resonanceCMR-was also executed 1 month after discharge).

\section{Discussion}

Despite the relatively benign course of Covid-19 in pediatric population, since

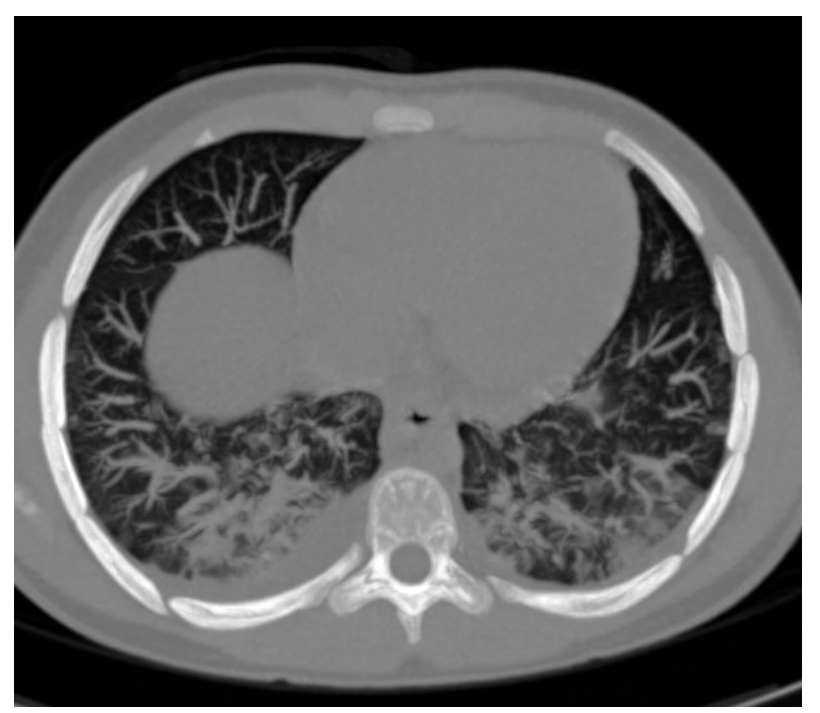

Figure 3. CT: Chest computed tomography image showing bilateral ground-glass opacities and pleural effusion. 
April 2020 there have been some reports of children with MIS-C and some cardiac involvement that often resemble features of Kawasaki disease, therefore most reported cases were treated with intravenous immunoglobulin, together with steroids [8] [9]. On the other hand there are several features that distinguish MIS-C, including prominent cardiac dysfunction with elevated BNPs. Our report describes SARS-CoV-2 related acute myocarditis in a pediatric patient, with no coronary damage. Pathophysiological underlying mechanism is currently uncertain.

It is known that angiotensin-converting enzyme 2 (ACE-2) is widely expressed in cardiomyocytes, cardiac fibroblasts and coronary endothelial cells and SARS-CoV-2 uses ACE-2 receptor for host cell entry. These observations might suggest that SARS-CoV-2 may directly cause myocardial injury. However, to our knowledge, there are no reports of SARS-CoV-2-RNA in the heart, to date [10].

The inflammatory activation with cytokines release syndrome, well documented in literature in COVID-19 adult patients and children, could also be considered as co-factor of heart damage [7] [8] [9] [10] [11]. The beneficial use of corticosteroids to attenuate inflammation is often used in SARS-CoV-2 critically ill adult patients [12] [13]. Such an inflammatory activation secondary to SARS-CoV-2 infection was also registered in our child. Steroid treatment indeed brought to a rapid clinical improvement in our patient, possibly confirming the key role of inflammatory cascade induced by SARS-CoV-2.

It is also known that excessive inflammation triggers platelet activation and endothelial dysfunction that may lead to thrombotic events, hence heparin was administered to our COVID-19 patient [14].

In our patient the onset symptoms were severe diarrhea and abdominal pain, which are reported in literature in $6 \%-8 \%$ of pediatric cases [5]; recent published data from adult series suggest a correlation between gastrointestinal symptoms at onset of COVID-19 and severe evolution of the disease [15] as observed in our child.

As far as concern antiviral therapy, given the severity of the clinical condition, we decided to start antiviral treatment for compassionate use with Remdesivir. Up to now there are no published papers focused on antiviral therapy with Remdesivir in SARS-CoV-2 infected children. However this drug demonstrated strength in trials related to other coronavirus infections. Although SARS-CoV and SARS-CoV-2 share only $82 \%$ RNA sequence identity, their RNA-dependent RNA polymerase (RdRp) shares $96 \%$ sequence identity. Therefore, Remdesivir targeting viral RdRp proteins of SARS-CoV is likely to be effective for SARS-CoV-2 [16].

\section{Conclusion}

Myocardial injury due to SARS-CoV-2 infection should be considered in pediatric COVID-19 severely symptomatic patients. There are still many unanswered questions concerning the pathophysiology of MIS-C and related cardiac involvement and optimal treatment. Antiviral treatment with Remdesivir in association 
with steroids could be effective in pediatric SARS-CoV-2 myocarditis.

\section{Limitations}

Despite CMR imaging represents the gold standard procedure in order to diagnose myocarditis [17], in this child it was not performed because it would have not changed the clinical management, moreover exposing healthcare staff to a further risk of infection [6]; CMR imaging was performed during outpatient follow-up for prognostic evaluation. Furthermore, endomyocardial biopsy was not performed because it is not routinely executed for myocarditis diagnosis in pediatric patients and it is usually performed in critical cases unresponsive to therapy [18].

\section{Acknowledgements}

Thank you to the patient's family for granting permission to publish this report.

\section{Conflicts of Interest}

The authors declare no conflicts of interest regarding the publication of this paper.

\section{References}

[1] Sohrabi, C., Asafi, Z., O’Neill, N., Khan, M., Kerwan, A., Al-Jabir, A., Iosifidis, C. and Agha, R. (2020) World Health Organization Declares Global Emergency: A Review of the 2019 Novel Coronavirus (COVID-19). International Journal of Surgery, 76, 71-76. https://doi.org/10.1016/j.ijsu.2020.02.034

[2] Wu, Z.Y. and McGoogan, J.M. (2020) Characteristics of and Important Lessons from the Coronavirus Disease 2019 (COVID-19) Outbreak in China: Summary of a Report of 72314 Cases from the Chinese Center for Disease Control and Prevention. JAMA, 323, 1239-1242. https://doi.org/10.1001/jama.2020.2648

[3] Huang, C.L., Wang, Y.M., Li, X.W., Ren, L.L. and Cao, B. (2020) Clinical Features of Patients Infected with 2019 Novel Coronavirus in Wuhan, China. The Lancet, 395, 497-506.

[4] Sinha, I.P., Harwood, R., Semple, M.G., et al. (2020) COVID-19 infection in children. The Lancet Respiratory Medicine, 8, 446-447. https://doi.org/10.1016/S2213-2600(20)30152-1

[5] Ludvigsson, J.F. (2020) Systematic Review of COVID-19 in Children Shows Milder Cases and a Better Prognosis than Adults. Acta Paediatrica, 109, 1088-1095.

https://doi.org/10.1111/apa.15270

[6] Hendren, N.S., Drazner, M.H., Bozkurt, B. and Cooper Jr., L.T. (2020) Description and Proposed Management of the Acute COVID-19 Cardiovascular Syndrome. Circulation, 141, 1903-1914. https://doi.org/10.1161/CIRCULATIONAHA.120.047349

[7] Jiang, L., Levin, M., Irfan, S., et al. (2020) COVD-19 and Multisystem Inflammatory Syndrome in Children and Adolescents. Lancet Infectious Diseases. https://doi.org/10.1016/S1473-3099(20)30651-4

[8] Verdoni, L., Mazza, A., Gervasoni, A., et al. (2020) An Outbreak of Severe Kawasaki-Like Disease at the Italian Epicenter of the SARS-CoV-2 Epidemic: An Observa- 
tional Cohort Study. The Lancet, 395, 1771-1778.

https://doi.org/10.1016/S0140-6736(20)31103-X

[9] Chiotos, K., Bassiri, H., Behrens, E.M., Blatz, A.M., Chang, J., Diorio, C., Fitzgerald, J.C., Topjian, A. and Odom John, A.R. (2020) Multisystem Inflammatory Syndrome in Children during the Coronavirus 2019 Pandemic: A Case Series. Journal of the Pediatric Infectious Diseases Society, 9, 393-398.

https://doi.org/10.1093/jpids/piaa069

[10] Guo, J.Y., Huang, Z., Lin, L. and Lv J.G. (2020) Coronavirus Disease 2019 (COVID19) and Cardiovascular Disease: A Viewpoint on the Potential Influence of Angiotensin-Converting Enzyme Inhibitors/Angiotensin Receptor Blockers on Onset and Severity of Severe Acute Respiratory Syndrome Coronavirus 2 Infection. Journal of the American Heart Association, 9, e016219.

https://doi.org/10.1161/JAHA.120.016219

[11] Qin, C., Zhou, L.Q., Hu, Z.W., et al. (2020) Dysregulation of Immune Response in Patients with COVID-19 in Wuhan, China. Clinical Infectious Diseases, 71, 762-768. https://doi.org/10.1093/cid/ciaa248

[12] Fu, Y.J., Cheng, Y.X. and Wu, Y.T. (2020) Understanding SARS-CoV-2-Mediated Inflammatory Responces: From Mechanisms to Potential therapeutics Tools. Virologica Sinica, 35, 266-271. https://doi.org/10.1007/s12250-020-00207-4

[13] The RECOVERY Collaborative Group (2020) Dexamethasone in Hospitalized Patients with COVID-19-Preliminary Report. N The New England Journal of Medicine. https://doi.org/10.1056/NEJMoa2021436

[14] Bikdeli, B., Madhavan, M.V., Jimenez, D., et al. (2020) COVID-19 and Thrombotic or Thromboembolic Disease: Implications for Prevention, Antithrombotic Therapy, and Follow-Up: JACC State-of-the-Art Review. Journal of the American College of Cardiology, 75, 2950-2973. https://doi.org/10.1016/j.jacc.2020.04.031

[15] Wan, Y.L., Li, J., Shen, L.H., et al. (2020) Enteric involvement in hospitalized patients with COVID-19 outside Wuhan. The Lancet Gastroenterology and Hepatology, 5, 534-535. https://doi.org/10.1016/j.jacc.2020.04.031

[16] Wang, M.L., Cao, R.Y., Zhang, L.K., et al. (2020) Remdesivir and Chloroquine Effectively Inhibit the Recently Emerged Novel Coronavirus (2019-nCoV) in Vitro. Cell Research, 30, 269-271. https://doi.org/10.1038/s41422-020-0282-0

[17] Ferreira, V.M., Schulz-Menger, J., Holmvang, G., et al. (2018) Cardiovascular Magnetic Resonance in Nonischemic Myocardial Inflammation: Experts Recommendations. Journal of the American College of Cardiology, 72, 3158-3176. https://doi.org/10.1016/j.jacc.2018.09.072

[18] Brighenti, M., Donti, A., Giulia Gagliardi, M., et al. (2016) Endomyocardial Biopsy Safety and Clinical Yield in Pediatric Myocarditis: An Italian Perspective. Catheterization and Cardiovascular Intervention, 87, 762-767.

https://doi.org/10.1002/ccd.26319 